\title{
Lehren, Lernen und Behandeln unter besonderen Umständen
}

Bruno Zeder

1 Lotus Children Projects, Schönau 1, 6332 Hagendorn, www.lotus-children.ch, info[at]lotus-children.ch

Korrespondenz:

Dr. med. Bruno Zeder Oberarzt INZ

Kantonsspital Aarau

Tellstrasse 5

CH-5001 Aarau

Tel. 0628384141

bruno.zeder[at]ksa.ch

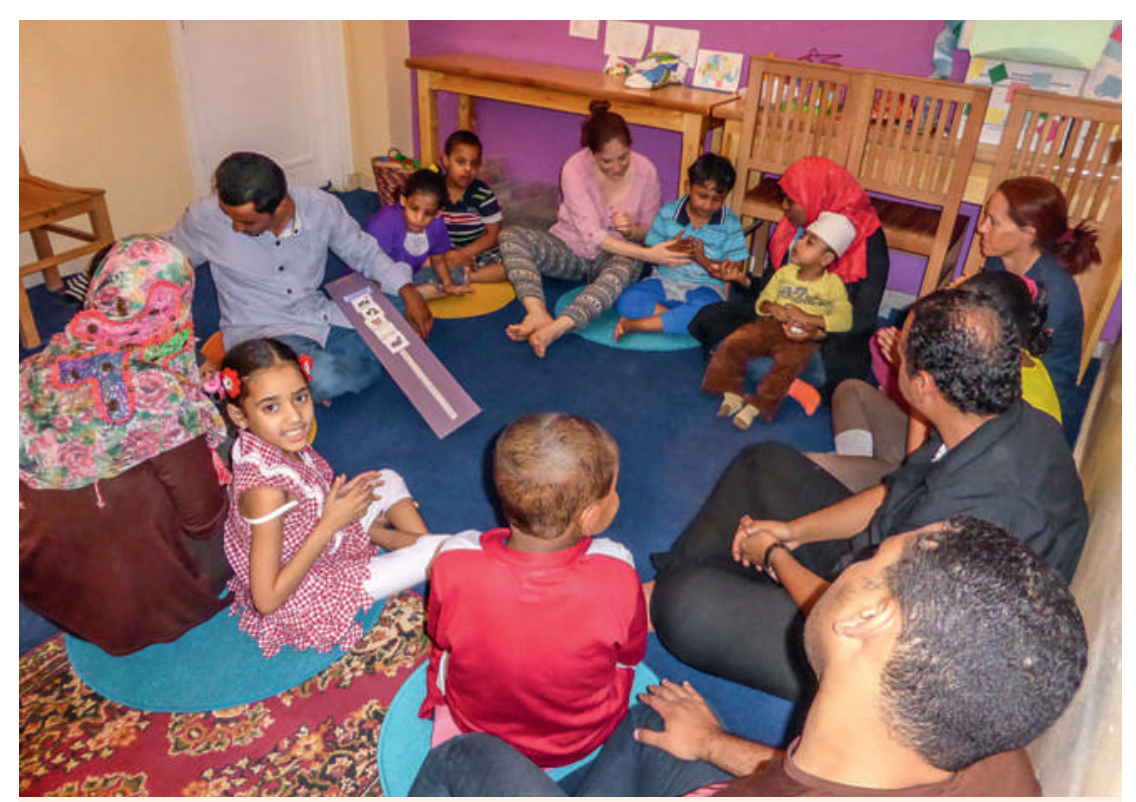

Jasmina Meier (rechts) und Nora Zeder (Mitte) mit den Kindern und Betreuungspersonen in der Morgenrunde.

Meine Tochter, Logopädin an der Heilpädagogischen Schule Zug, wurde diesen Frühling zu einem Teaching nach Aswan, Ägypten eingeladen, wo die Heilpädagogin Jasmina Meier vor drei Jahren ein Projekt [1] startete, mit dem sie sich schon viele Jahre beschäftigt: dem Aufbau einer eigenen Schule für geistig behinderte Kinder. Wahrlich eine gewaltige Herausforderung in einem Land, das von Krisen, Gewalt und Korruption beherrscht wird.

Die Mutter von Jasmina Meier, eine pensionierte Kindergärtnerin, hat in Aswan seit 12 Jahren einen Zweitwohnsitz, kennt Land und Leute bestens und ist mit deren Kultur vertraut, wohl eine der wichtigsten Voraussetzungen für ein solches Vorhaben. Zusammen mit ihrem Partner rief Jasmina Meier eine Stiftung ins Leben. Mit grossem persönlichen Einsatz und bescheidenen Mitteln hat sie schon viel erreicht. Erst vor wenigen Wochen erhielt sie eine Arbeitsbewilligung und kann die Schule, die sie zuvor über eine einheimische Organisation aufbaute und betrieb, nun selber führen. Anfang November 2014 findet in den neuen Räumlichkeiten die Eröffnung statt.

\section{Andere Sitten in der Hadana}

Bei den Vorbereitungen meiner Tochter zeigt sich, dass auch der Einsatz eines Arztes sinnvoll sein könnte, und so entschloss ich mich, sie zu begleiten.
Mit viel Vorfreude und Koffern voller Spielsachen reisten wir nach Ostern ins oberägyptische Aswan und trafen in einer bescheidenen, aber herzlich eingerichteten Wohnung auf ein kleines Paradies. Zehn Kinder im Alter von 4 bis 14 Jahren wurden damals dort betreut, deren Behinderungsspektrum reichte von einem Kind mit Downsyndrom über Kinder mit CP, Entwicklungsverzögerungen und komplexen hirnorganischen Störungen bis zu Autismus, alle mit schweren Sprachentwicklungsstörungen und sozialen Problemen. Das Team bestand aus vier einheimischen Lehrkräften, alle mit einer pädagogischen Ausbildung, wobei man diese nicht mit unseren Vorstellungen messen darf. Jasmina Meier hat sich der grossen Aufgabe gestellt, diese Lehrer auszubilden und zu einer Gruppe zu formen.

Der Tagesablauf in der Hadana (ägyptisch für «Kindergarten») ist klar strukturiert und gibt den Kindern den Rahmen zur Entfaltung und gezielten therapeutischen Arbeit, ist jedoch auch nötig, um das Lehrerteam zu führen und kontinuierlich auszubilden, ist doch ein therapeutisches Konzept dort gänzlich unbekannt. In den öffentlichen Schulen (Klassen mit über 40 Kindern sind die Regel), aber auch in privaten Unterrichtsstätten wird nach einhelliger Aussage der Lehrer und Eltern ein ausschliesslich direktiver Unterricht erteilt, die Kinder werden uniform ausgebildet, der Erziehungsstil ist rau, bis hin zu körperlicher Züchtigung. Logopädie wird an den öffentlichen Schulen von spezialisierten Lehrpersonen erteilt, die den Kindern dauernd ins Gesicht greifen, ein Verhalten, das auch bei den Lehrern an der Hadana immer wieder beobachtet werden konnte und von Jasmina Meier sofort korrigiert wurde. Der liebe- und respektvolle Umgang mit den Kindern ist das Ziel der Bemühungen von Jasmina Meier, es stellt die Basis des therapeutischen Handelns dar. Was so einfach tönt, ist in der Umsetzung eine riesige Herausforderung, da die sozialen und kulturellen Voraussetzungen dafür erst in Ansätzen vorhanden sind, jedoch von den Eltern sehr geschätzt werden.

\section{Strukturierter Tagesablauf und Massagen für die Kinder}

Die Kinder werden in der Morgenrunde zusammengenommen, mit Gesang und Piktogrammen wird das Tagesprogramm eingeleitet und das Wochenthema besprochen. Es folgen Gruppen- und Einzelunterricht, auch die Trink- und die Mittagspause 


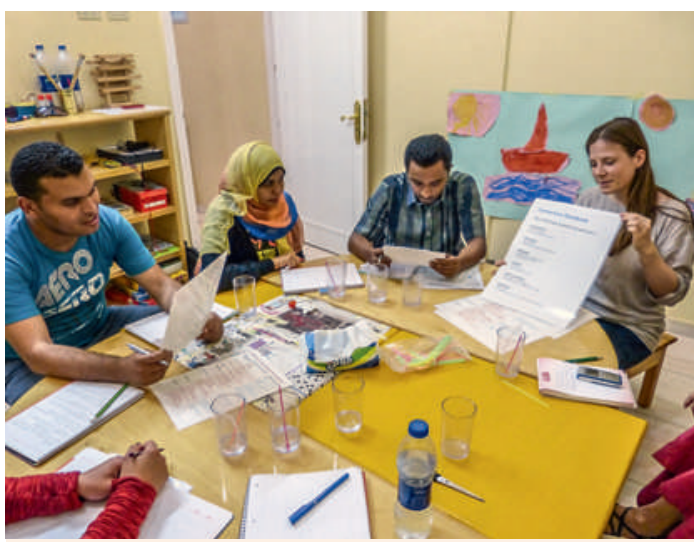

Nora Zeder beim Teacher Training.

werden pädagogisch genutzt. In der anschliessenden Ruhephase steht den Kindern eine Matratze zum Liegen zur Verfügung, sie werden in dieser Zeit von den Lehrern an Kopf und Füssen massiert und geniessen diese Zuwendung enorm, ebenso auch die Hintergrundmusik. Am Nachmittag wird dann nochmals intensiv in der Gruppe gearbeitet. Beim Basteln mit grob- und feinmotorischen Übungen und Sprachförderung wird besonderes Gewicht auf alltagsrelevante behinderungsangepasste Aspekte gelegt. Jeder Situation wird von Jasmina Meier ein pädagogischer Hintergrund unterlegt, eine grosse Herausforderung für die Lehrer, die einen kindergerechten Umgang erlernen müssen. Die kulturellen Besonderheiten werden besonders berücksichtigt, leben doch in Aswan neben den Muslimen auch eine Minderheit koptischer Christen (Nubier), deren Islamisierung derzeit besonders stark betrieben wird.

\section{Ärztlicher Rat aus der Schweiz ist gefragt}

In einem Kontaktheft, ebenfalls ein Novum dortzulande, werden den Eltern Informationen gegeben, ebenso besteht aber bei der Verabschiedung immer auch die Möglichkeit zu einem direkten Austausch. So hatten wir Gelegenheit zu eigentlichen Elterngesprächen, in denen Fragen zu Erziehung, Sprachförderung, Ernährung und Übungen zu Hause besprochen wurden. Rasch sprach sich unsere Anwesenheit herum und so suchten Eltern, deren Kinder nicht in der Hadana betreut werden, ebenso auch Erwachsene mit Gesundheitsstörungen Rat. So wurde mir unter anderen ein vier Monate altes Kind mit einer wohl seit der Geburt bestehenden und bisher verkannten pronation douloureuse zur Behandlung gebracht.

\section{Teacher Training hat hohen Stellenwert}

2 Portman A. Wenn mir die Worte fehlen. Luzern: Verein Projekt «Wenn mir die Worte fehlen»; 2012

3 Evangelical Mission Hospital. Al Germaniah, Aswan. Chefarzt Dr. H. Bahaa, bahaahanna[at]me.com
Besonderes Gewicht wurde auf das Teacher Training gelegt. Einerseits direkt während des ganzen Tages durch unmittelbares Ausbilden, Instruieren, Korrigieren und Üben, auch mit Einsatz der Gebärdensprache (nach Anita Portmann) [2]. Andererseits vermittelte meine Tochter in den Teamsitzungen eine Einführung in die Sprachentwicklung, die ja unab- hängig von der jeweiligen Muttersprache verläuft. Ebenso stellte sie das Sprachförderungskonzept des «Corrective Feedback» mit konkreten Übungen und Umsetzung im Alltag vor.

Wir hatten Gelegenheit, das Germania-Hospital in Aswan [3] zu besuchen. Dieses Spital wurde vor 100 Jahren von deutschen und schweizerischen evangelischen Missionaren gegründet und wird heute von einem Ägypter (mit einer Deutschen verheiratet) geleitet. Aus der Not heraus wurde dort vor zwei Jahren für die schwerstbehinderten Kinder eine Tagesstätte aufgebaut. Auch hier werden ausländische Fachkräfte für die Ausbildung des Personals beigezogen (wie übrigens auch im Arztbereich des Spitals). Die Gattin des Chefarztes leitet diese Gruppe nebenbei. Zwei Tage wurden wir auch hier um Ausbildung und Schulung des Personals gebeten, die grosse Wissbegier und das sofortige Umsetzen überraschte uns. Die Kommunikation bot wenig Schwierigkeiten und klappte mit Deutsch und Englisch und direkter Übersetzung. Auch wurde ich vom Chefarzt gebeten, ihn selber wegen seiner chronischen Kopfschmerzen zu untersuchen und manualtherapeutisch-osteopathisch zu behandeln, eine dort gänzlich unbekannte Technik.

\section{Wenig Früchte und Gemüse, viel Fast Food und tiefe Löhne}

Natürlich hatten wir auch Zeit, das wunderschöne Aswan zu besuchen, die Museen und Ausgrabungsstätten. Dank der Verbindungen von Jasmina Meier kam es zu vielen Kontakten mit den Einheimischen, wobei uns vor allem auch die Freundlichkeit und noble, zurückhaltende Art der Nubier auffiel.

Beim Gang durch den Markt fiel auf, wie wenig Früchte und Gemüse angeboten wurden, ganz im Gegensatz zum allgegenwärtigen Fast Food. Das Essen im Souk ist günstig, ein schmackhaftes Gericht mit Salat und Eintopf kostet 40 Rappen. Traurig stimmte der Umstand, dass die Menschen, die vom Tourismus leben, am verarmen sind. Sie versuchen alle Mögliche zu verkaufen, um etwas Geld zu verdienen, laut Einheimischen wird jetzt auch immer mehr der Familienschmuck veräussert.

So ist denn verständlich, dass für die Menschen dort ein Job enorm viel bedeutet. Die Löhne sind tief. Der Mindestjahreslohn im öffentlichen Dienst beträgt derzeit ca. 500 ägyptische Pfund, entsprechend 60 Franken. Das Jahres-Budget der Lotusstiftung beläuft sich auf 20000 Franken, alles aus Spenden der Mitglieder. Für die Betreuung eines Kindes in der Hadana bezahlen die Eltern monatlich fünf Franken, gegebenenfalls wird das Kind auch ohne Entgelt aufgenommen. Der Arbeit von Jasmina Meier und ihrem Team gebührt unser ganzer Respekt, verbunden mit der Hoffnung, dass sie nun mit der eigenständigen Struktur und in den neuen Räumlichkeiten im Zentrum auch die Zielsetzungen verwirklichen kann. 